\title{
Nanotechnologies in Glycoproteomics
}

\author{
Hu Zhao ${ }^{1}$, Yaojun $\mathrm{Li}^{1}$ and $\mathrm{Ye} \mathrm{Hu} \mathrm{H}^{1,2^{*}}$
}

\begin{abstract}
Protein glycosylation, as an important post-translational modification, is implicated in a number of ailments. Applying proteomic approaches, including mass spectrometry (MS) analyses that have played a significant role in biomarker detection and early diagnosis of diseases, to the study of glycoproteins or glycopeptides will facilitate a deeper understanding of many physiological functions and biological pathways involved in cancer, inflammatory and degenerative diseases. The abundance of glycopeptides and their ionization potential are relatively lower compared to those of non-glycopeptides; therefore, sample enrichment is necessary for glycopeptides prior to MS analysis. The application of nanotechnology in the past decade has been rapidly penetrating into many diverse scientific research disciplines. Particularly in what we now refer to as the "glycoproteomics area", nanotechnologies have enabled enhanced sensitivity and specificity of glycopeptide detection in complex biological fluids, which are critical for disease diagnosis and monitoring. In this review, we highlight some recent studies that combine the capabilities of specific nanotechnologies with the comprehensive features of glycoproteomics. In particular, we focus on the ways in which nanotechnology has facilitated the detection of glycopeptides in complex biological samples and enhanced their characterization by MS, in terms of intensity and resolution. These studies reveal an increasingly important role for nanotechnology in helping to overcome certain technical challenges in biomarker discovery, in general, and glycoproteomics research, in particular.
\end{abstract}

\section{Introduction}

Protein glycosylation is one of the most common posttranslational modifications (PTMs) in living cells [1,2]. Glycoproteins play a vital role in biological processes including cell adhesion, receptor activation, and signal transduction [3-5]. As such, altered or erroneous glycosylation is often associated with inflammatory diseases, neurodegenerative disorders, and even certain cancers [6,7]. For example, several investigators have observed that the abnormal expression of different glycosylated proteins were implicated in disease and that changes in the level of glycoprotein could be used as hallmarks for disease diagnosis, including the carbohydrate antigen CA19-9 for colon cancer [8], the prostate-specific antigen (PSA) for prostate cancer [9], $\alpha$-fetoprotein for liver cancer [10], and $\beta$-human chorionic gonadotropin for germ cell tumors [11]. Furthermore, many accessible, membranebound or extracellular proteins are glycosylated and could

\footnotetext{
* Correspondence: yhu@houstonmethodist.org

'Department of Nanomedicine, Houston Methodist Research Institute, Houston, TX 77030, USA

${ }^{2}$ Department of Cell and Developmental Biology, Weill Cornell Medical College, New York City, NY 10021, USA
}

be exploited for therapeutic intent, such as the Her2 receptor for breast cancer therapy [12].

Thus far, analytical methods of identifying (sometimes subtle) changes in disease-relevant glycoproteins can be divided into two main groups: glycoprotein- or glycopeptidebased analysis. We illustrate the workflow of these two approaches in Figure 1 [1]. In the first (glycoprotein-based) approach, glycoproteins are enriched by different separation methods, such as size exclusion, ion exchange, affinity chromatography, chemical immobilization, and other methods. To identify the protein part of a glycoprotein is much easier than to identify the glycan part of the same glycoprotein. In the latter (glycopeptide-based) strategy, the glycoproteins initially undergo enzymatic or chemical degradation, and the resulting glycopeptides can be enriched by several methods, such as lectin-affinity chromatography [13-15], boronic acid-based approach [16,17], hydrazide chemistry [18-20], or solid-phase extraction using hydrophilic interactions [21]. The enriched glycopeptides are then deglycosylated and quantified via MS analysis. Consequently, the sequences of various glycopeptides and their specific glycosylation sites can be easily identified. With the advent of ever more sophisticated MS modalities, we can now delve even deeper in the realm of glycoproteomics research. Still, 


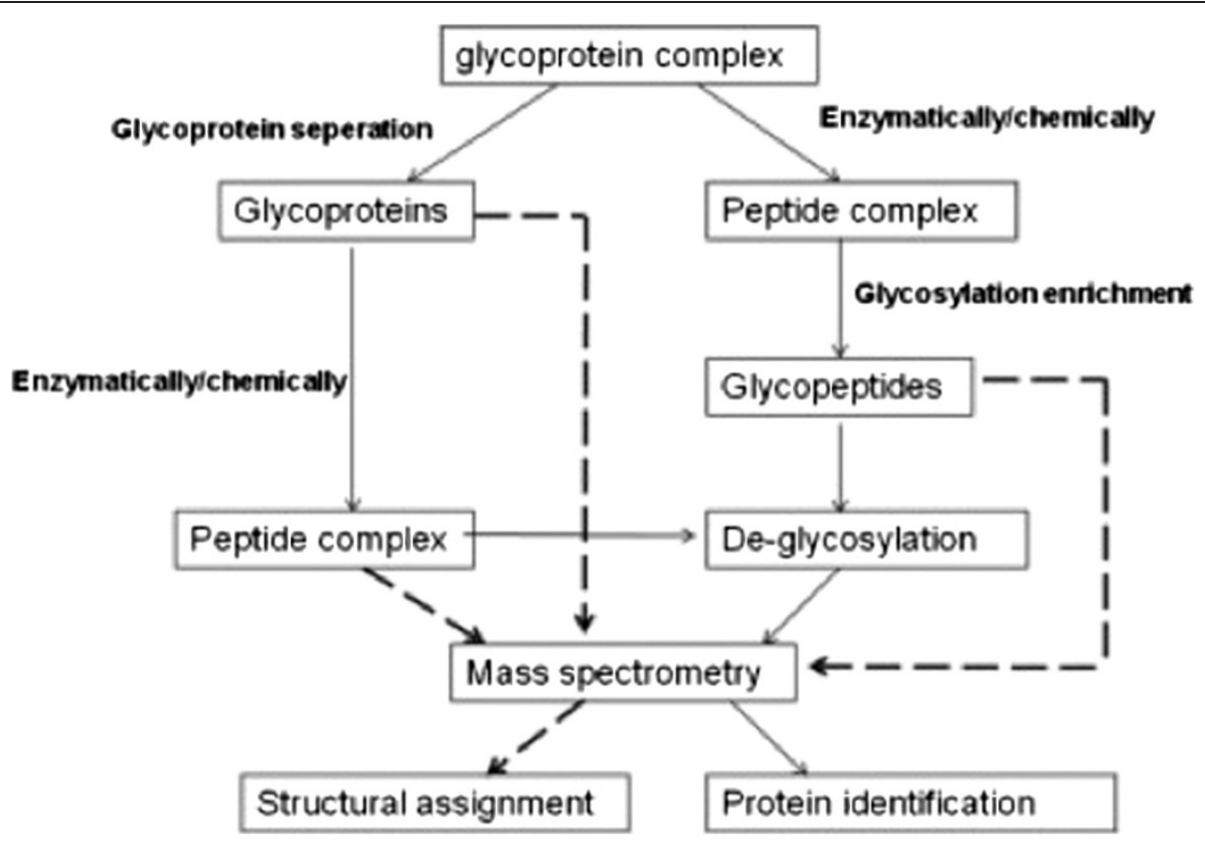

Figure 1 Typical workflow for glycoproteomics analyses [1].

challenges remain for profiling glycopeptides in complex samples (e.g., serum or blood) using current MS-based techniques, because the proportion of glycopeptides in total solution is quite small, and their signals in MS profiles are often obscured by those of non-glycosylated peptides [22]. Therefore, enhancing the specificity and sensitivity of glycopeptide enrichment and detection in complex samples remains a very real challenge.

In the past decade, we have witnessed an enormous growth in the application of nanotechnology to solve biological and biomedical problems. Due to some of these (nano) technological advances, we can now identify and analyze biomolecules at a speed and resolution never seen before. Compared to conventional macro-scale materials, nano materials, such as nanoparticles, nanowires, nanotubes, nanorods, and thin films with meso-scale pores, have unique transport properties (i.e., more efficient electron transport), better optical excitation and high detection efficiency. Nanomaterials also impart to their fabricated platforms higher surface-to-volume ratio with reduced dimensionality, which are key features that often enhance the physical and chemical properties of materials. In addition, materials with lower dimensionality are conducive to extremely high unit-density integration on arrays and lab-on-a-chip platforms for point-of-care devices for high-throughput biodetection. Miniaturized devices composed of nanomaterials have the advantage of low cost, good portability, and potential use in minimally invasive instrumentation [23]. In Figure 2, we present some examples of nanomaterial-enabled platforms for biomolecule detection, such as silicon nanowire field-effect transistor (SiNW-FET) sensors for detecting prostatespecific antigen (PSA), Real-time Quartz Crystal Microbalance $(\mathrm{QCM})$ for detecting serum proteins and $\mathrm{ZnO}$ nanorod platforms for detecting protein interaction. We and others have developed a series of mesoporous silica thin film chips with a variety of nanotextures that can selectively enrich for low molecular weight peptides from complex biological fluids, as part of a biomarker discovery platform [24-28].

Glycoproteins are not as amenable to isolation and identification due confounding factors such as complex glycan structures, glycan isomers, and amino acid residues attached to the oligosaccharide chains. The most commonly employed technique for the analysis of protein glycosylation (glycosylation site identification, glycan structure determination, site occupancy, and glycan isoform distribution) is MS, albeit with still a few existing technical challenges [32]. In this review, we will highlight some of the platforms that have been developed for the enrichment of glycopeptides from complex samples, and introduce emerging combination platforms that couple the sensitivity and accuracy of MS to the exquisite selectivity of nano-platforms.

\section{Different nano-platforms used to enrich glycopeptides}

A nanomaterial is a natural, incidental or manufactured material containing particles in an unbound, aggregate or agglomerate state, where one or more external dimensions is in the size range 1-100 nanometers. Due to their smaller size, nanomaterials possess incredibly larger surface areas 
(a)
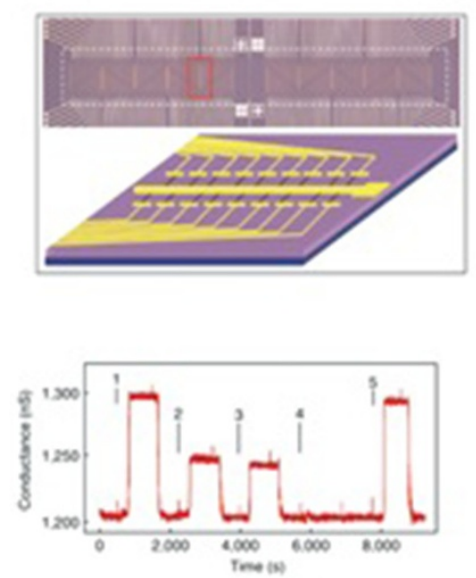
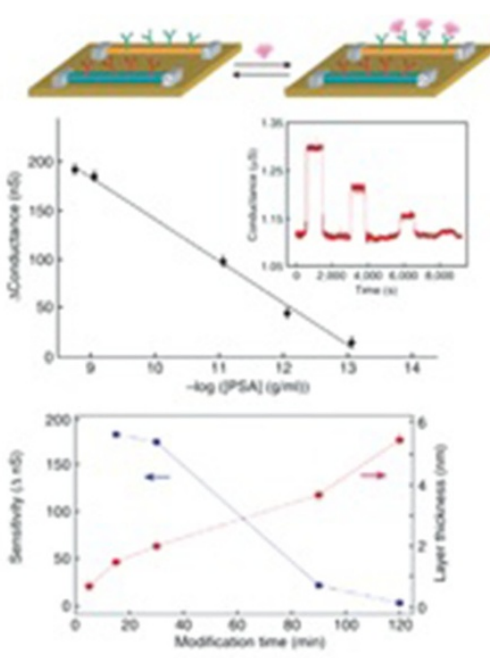

(b)
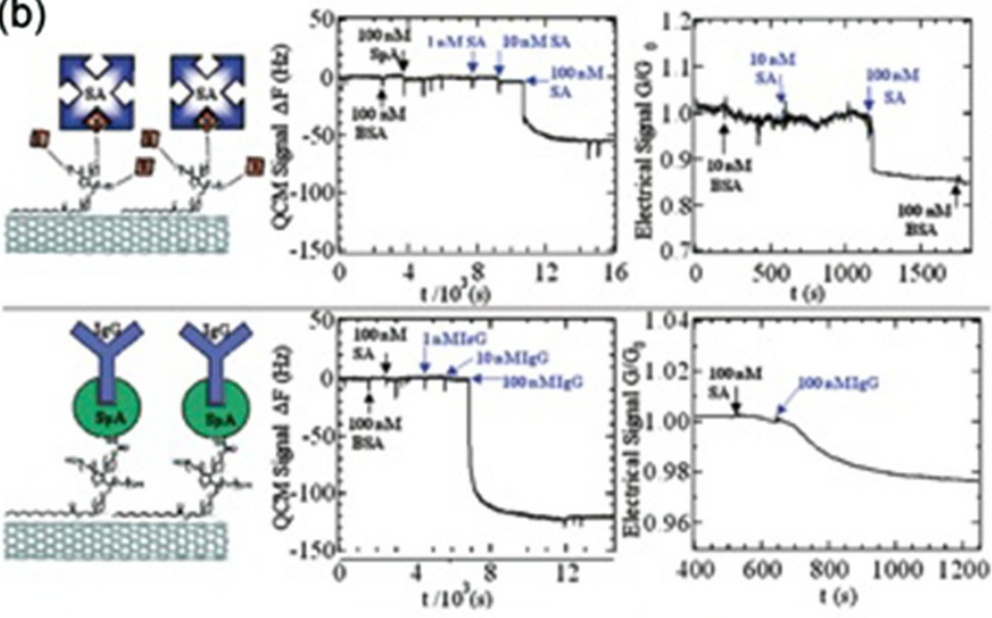

(c)
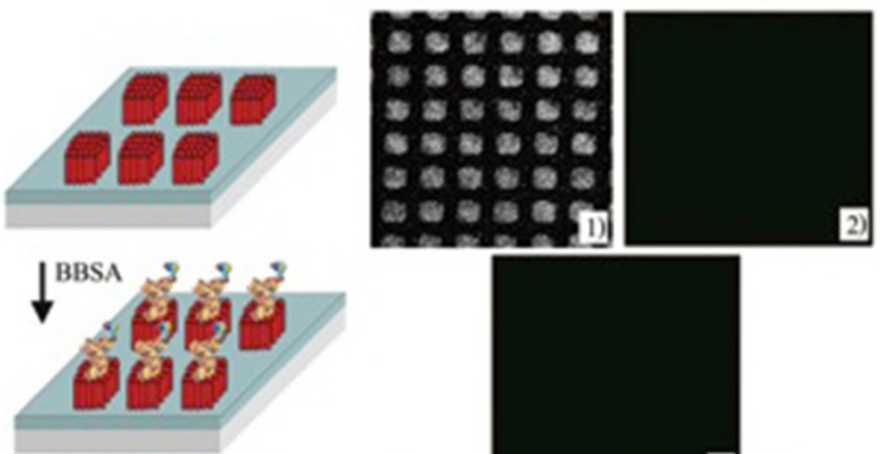

| DTtreptavidig of of
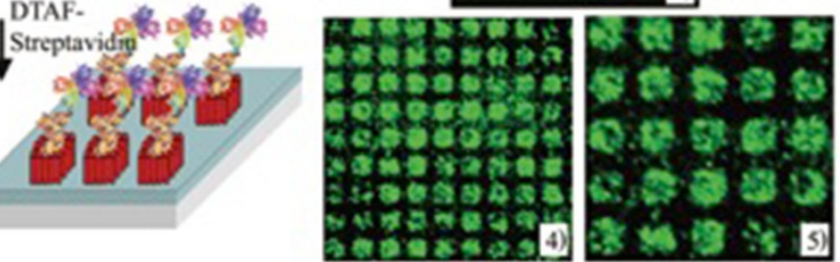

Figure $\mathbf{2}$ (See legend on next page.) 
(See figure on previous page.)

Figure 2 Examples of nano-dimensioned biosensors. (a) SiNW FET sensors are used to detect prostate-specific antigen (PSA) [29]; (b) Real-time Quartz Crystal Microbalance (QCM) and electrical sensing of model proteins [30]; and (c) Enhanced fluorescence detection carried out on various biological systems coupled to ZnO nanorod platforms. Scanning electron microscopy (panel 1) and fluorescence (panels 2 through 5) images of biotinylated bovine serum albumin (bBSA) and fluorescein derivativeconjugated streptavidin (DTAF-strept) on ZnO nanorods [31].

than bulky materials. This feature provides more activated sites for glycopeptide enrichment and unique optical properties, in the context of rapid and sensitive detection. Perhaps the most attractive characteristic of nanomaterials is the degree to which their size, shape and morphology can be precisely controlled to enhance the optical, electronic, or magnetic capabilities typically desired in certain applications. Several nano-platforms exist for the isolation of glycopeptides in complex samples with improved sensitivity and specificity, and we discuss them in more details in the following Subsections.

\section{Nanoparticles for glycopeptide enrichment}

Nanoparticles are arguably the most widely used nanomaterial to assemble chips for biomolecule enrichment because: 1) nanoparticles can be synthesized with various size distributions, and morphologies; 2) nanoparticles can be modified and diversified by numerous reagents for specific uses; and 3) they exhibit unique optical and electrochemical features. When attempting to enrich glycopeptides in complex samples, researchers often adopt core-shell structured nanoparticles with a magnetic core within a thin layer of compatible material. The magnetic core, which provides a strong magnetic force, would be simply attracted by magnets and the outer layer would be easily modified by various organic or inorganic reagents to recognize the biomarker of interest.

Using a version of the above approach, Zhou et al. fabricated aminophenyl boronic acid-functionalized magnetic nanoparticles and used them to selectively capture glycopeptides and glycoproteins from mixtures containing nonglycomoleculars [33]. Other groups have also applied versions of surface-modified nanoparticles for glycoprotein selection. For example, 3-aminopropyltriethoxysilane (APTES) is frequently used as a surface-modifying reagent for silica nanoparticles, whereby the amino group imparts a positive charge. Zhang Y. and coworkers designed a one-step salinization reaction to assemble APTES onto the nanoparticle surface in order to capture aldehyde groups on oxidized glycopeptides. These nanoparticles greatly reduced the coupling time from 12-16 hours to just 4 hours without sacrificing enrichment efficiency, compared to traditional solid-phase extraction methods based on hydrazide resins [34]. Zhang L. et al. developed a novel composite material with a core-satellite structure. These are gold nanoparticles, functionalized with boronic acid, and then anchored on the surface of the silica-coated magnetic core. Using this strategy, the investigators were able to recover $85.9 \%$ of glycopeptides and $71.6 \%$ of glycoproteins after enrichment. The composite nanoparticles had an adsorption capacity of more than $79 \mathrm{mg}$ of glycoproteins per gram of the material. The investigators used these new composite nanoparticles to enrich glycosylated proteins from human colorectal cancer tissues for subsequent identification of N-glycosylation sites. They were able to map 194 unique glycosylation sites, of which 165 sites (85.1\%) were newly identified, to 155 different glycoproteins [35].

Polysaccharides can also be used as a surface-modifying component for glycopeptide enrichment. Xiong et al. reported a layer-by-layer approach to synthesize magnetic nanoparticles (MNPs) that are coated with multilayered polysaccharide shells, and then used these hydrophilic materials for selective enrichment of glycopeptides from biological samples. These investigators identified 605 unique $\mathrm{N}$-glycosylation sites in 616 distinct glycopeptides, corresponding to 350 glycosylated proteins in $20 \mu \mathrm{g}$ mouse liver protein sample. Their results indicated that exploiting specific sugar-sugar interactions shows promise as a design strategy for characterizing protein glycosylation [36].

Rather than using boronic acid as a surface-modifying reagent for nanoparticles, Tran et al. developed a new kind of gold nanoparticles, functionalized with ultrasmall hydrazide groups, with a core diameter of $1.2 \mathrm{~nm}$. The assembly process involves an oxidation step and covalent coupling, whereby the carbohydrate moiety of the glycoproteins are oxidized into aldehydes by periodate and the oxidized glycoproteins are covalently coupled to hydrazide resin. No glycoproteins are removed by washing. They successfully utilized these nanoparticles to isolate $90 \%$ of the glycopeptides from complex biological samples. The nanoparticle stability in biological solution, unique solubility, and large capacity for peptide capturing provide great application potential in glycoproteomics studies [37].

Isolating glycopeptides via their lectin moiety is another common approach, and commercialized solutions are now available in the market. Tsutsumi and colleagues conjugated gold nanoparticles (GNPs) with monosaccharidemodified peptides as optical probes for lectin detection. The aggregation of the glycopeptide-modified GNPs with concanavalin A (ConA) causes an absorption shift from $534 \mathrm{~nm}$ to $620 \mathrm{~nm}$, an apparent color change that can be detected by the naked eye [38].

Ligand-modified nanoparticles have been proven to be an excellent platform for glycopeptide enrichment, but ligand-free nanoparticles are emerging as good candidates 

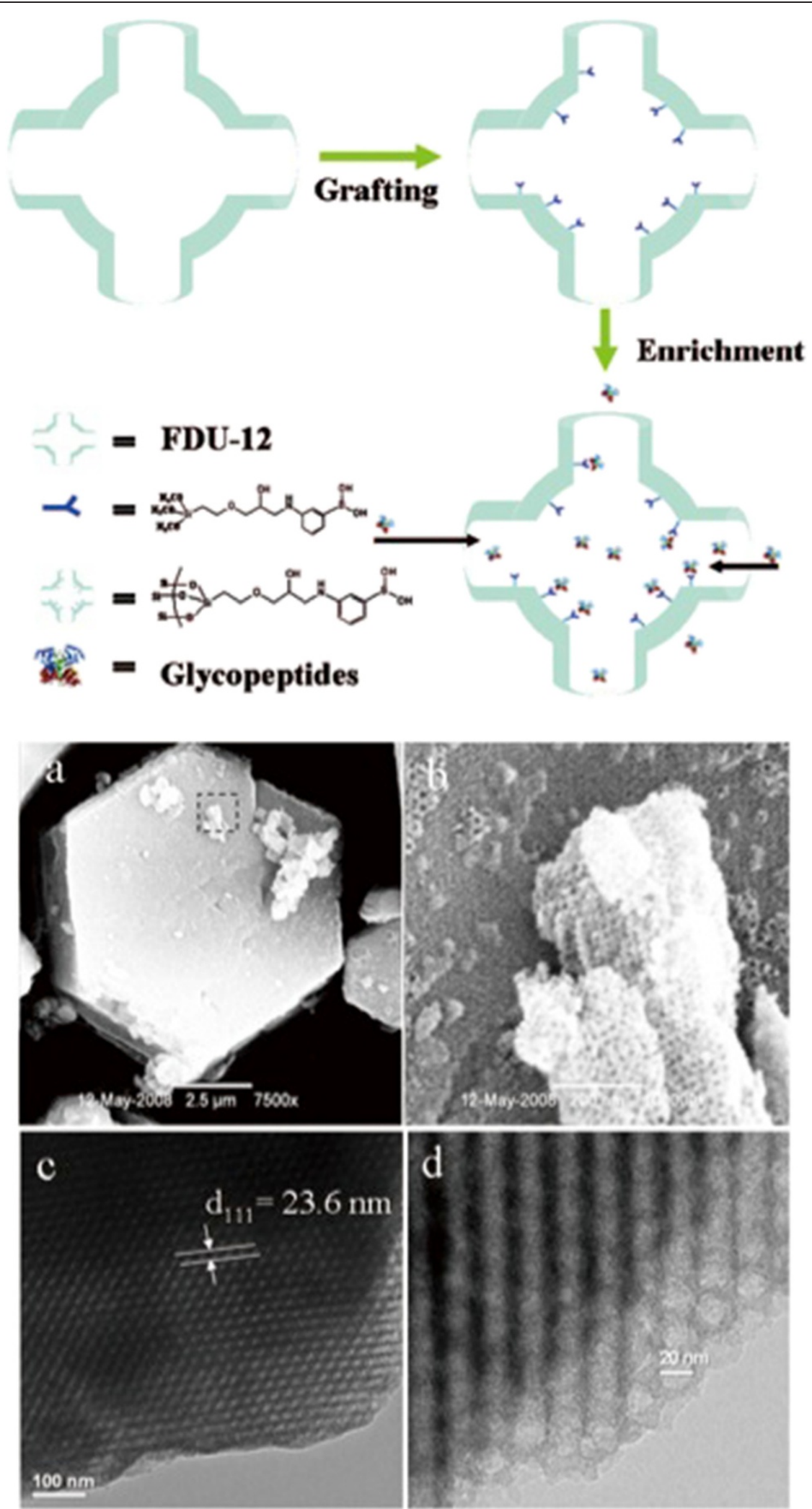

Figure $\mathbf{3}$ (See legend on next page.) 
(See figure on previous page.)

Figure 3 A schematic of glycopeptide enrichment from dilute solutions using mesoporous silica material modified with boronic acid.

The pores act as a nanoreactor for the specific binding of di-boronic acid groups to glycopeptides (top half of the figure). FE-SEM images of

boronic acid-modified mesoporous silica material at low magnification (a) and the high-resolution image of the squared area in part a; and

(b) TEM images along the vertical direction of the mesoporous materials (c,d) [41].

for glycoproteins or glycopeptides isolation. A research group in Fudan University developed ligand-free silver nanoparticles coated with magnetic nanoarchitecture for selective enrichment of glycopeptides by taking advantage of the reversible interaction of glycans with silver nanoparticles. The investigators could easily and quickly (only 1 min incubation time) extract glycopeptides at a low molar ratio of glycopeptides: non-glycopeptides (1:100) using these silver-nanoparticle magnetic beads. Moreover, they mapped 127 unique glycopeptides mapped to 51 different glycoproteins from a very small sample volume (only $1 \mu \mathrm{L}$ rat serum) [39].

Particle-based materials are the most widely adopted for isolating and separating glycosylated peptides due to their nano dimensionality, tightly controlled size, and unique optical and electrochemical properties. They will likely continue to play a prominent role in glycoproteomics for biomarker detection and early diagnosis of diseases.

\section{Mesoporous materials for glycopeptide enrichment}

Several classes of porous materials exist and group according to size. The International Union of Pure and Applied Chemistry (IUPAC) clarifies [40], microporous materials and macroporous materials as having pore diameters less than $2 \mathrm{~nm}$ and greater than $50 \mathrm{~nm}$, respectively; mesoporous materials place in the middle. Developed since the 1970's, mesoporous silica materials are, at present, widely used in drug delivery, imaging, biosensors, for example, owing to their regular pore arrangement, simple preparatory methods, and low cost. Under the influence of strong capillary forces, biomolecules entering the mesoporous pores encounter more activation sites for binding, resulting in higher separation efficiency. Many glycoproteomics studies have been conducted using mesoporous materials for glycopeptide isolation and separation.

$\mathrm{Xu}$ and colleagues first introduced mesoporous silica materials functionalized with boronic acid for the detection of glycosylated peptides. In Figure 3, we illustrate the basic workflow of this approach. The field emission electron scanning microscope images of the prepared products show a pattern of regular honeycomb-like hexagonalpatterned pores. Glycopeptides in solution bound with high affinity to the groups attached within the pores, markedly improving the solute detection limit [41]. Similarly, Liu et al. [22] and Zhang H. et al. [42] applied mesoporous silica functionalized with aminophenylboronic acid (APB) to analyze glycopeptides in standard protein solutions and rat serum, respectively.

Other than boronic acid, transition metal oxides can be coupled to mesoporous silica, as demonstrated by Wan and coworkers. These investigators coated mesoporous silica microspheres in a layer of zirconium dioxide to create a platform for hydrophilic interaction liquid chromatography (HILIC) solid phase extraction (SPE) (depicted in Figure 4). HILIC has broad glycan specificity, good reproducibility, and compatibility with $\mathrm{MS}$ analysis. $\mathrm{ZrO}_{2}$ species were highly dispersed on the surface of mesostructured cellular foam. Hydrogen bonding between the oxygen atoms of $\mathrm{ZrO}_{2}$ and the hydrogen atoms of glycans forms a relatively strong interaction, thus isolating the glycopeptides from complex samples. Since $\mathrm{ZrO}_{2}$ can also attract phosphopeptides, the investigators treated digested mixtures of the phosphoprotein, $\alpha$-casein, and IgG with $\mathrm{ZrO}_{2} /$ MPS HILIC SPE materials. The results demonstrated that glycopeptides could be effectively enriched with interference from Zirconia layer coated silica surface [43].

Oxidized mesoporous carbon is another very useful material in glycoproteomics research because of its extraordinary hydrophilicity. Hence, oligosaccharides on N-linked glycans interact with the carbon functional groups, enabling glycopeptide separation prior to profiling by MS. Using this approach, Qin et al. enriched N-linked glycans using oxidized and ordered mesoporous carbon materials of CMK-3 before matrix-assisted laser desorption/ionization time-of-flight (MALDI-TOF) MS. Twenty four N-linked glycans derived from standard glycoproteins could be detected with high signal-to-noise $(\mathrm{S} / \mathrm{N})$ ratios and peak intensities. Thirty-two N-linked glycans were profiled in complex samples, five (4 core-fucosylated glycans) of which exhibited distinct patterns in liver cancer compared to healthy samples. Their results support the notion that precisely-sized mesoporous carbon materials could play a prominent role in clinical glycoproteomics [44].

With such broad applicability and adaptability, we expect the use of mesoporous materials to continue leaving an impact on the glycoproteomics landscape. The large specific surface area, surface modification potential, size exclusion toward large molecular weight biomolecules, and support strong capillary force in nano scale are all characteristics that lend well to their wide-spread use.

\section{Other nano platforms for glycopeptide enrichment}

Two-dimensional (2D) crystalline materials have recently been identified and analyzed for use in glycopeptide 


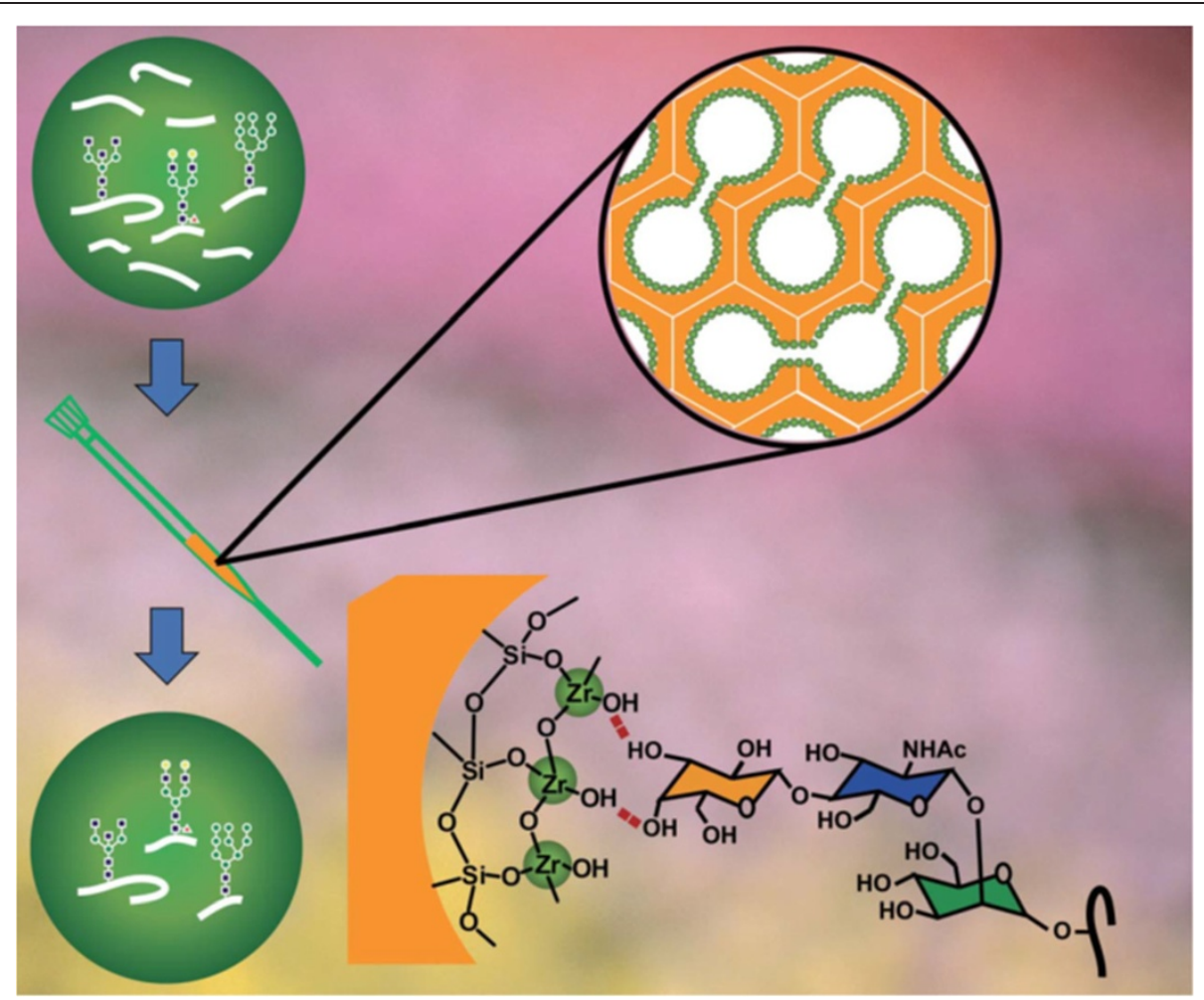

Figure 4 A schematic representation of glycopeptide isolation using mesoporous silica microspheres coated with zirconia [43].
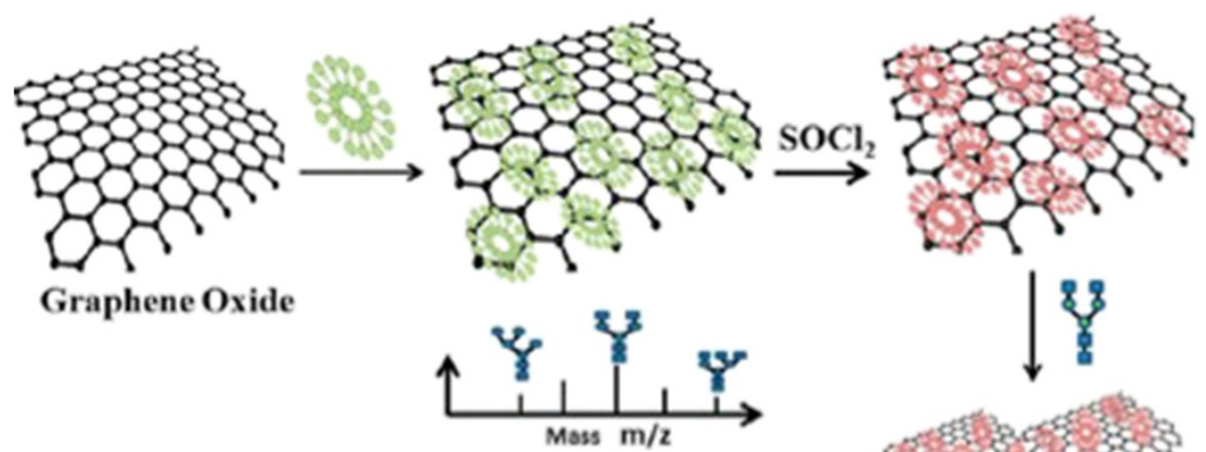

Graphene Oxide
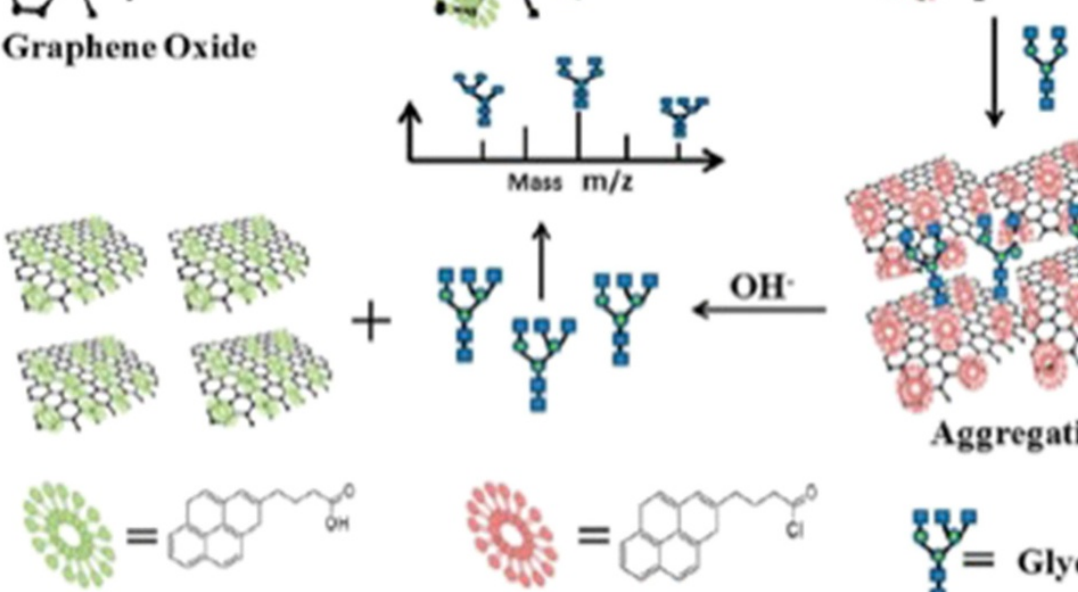

1-pyrenebutyric acid
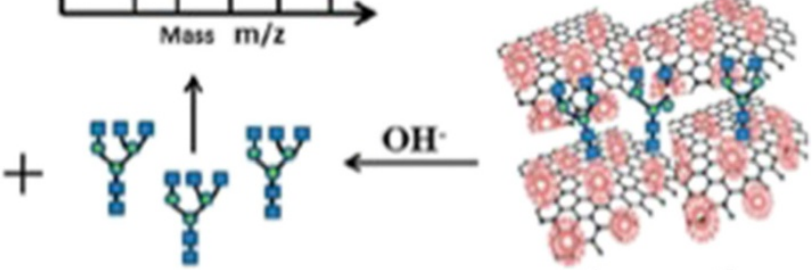

\section{Aggregation}
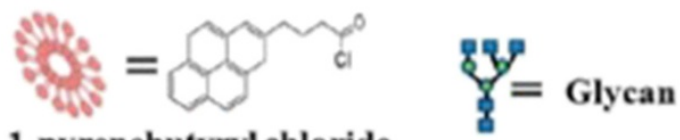

Figure 5 Using functionalized graphene for glycan separation [46]. 
enrichment [45]. Graphene, consisting of a single atomic layer of carbon, is the first applied in this new class of materials. A number of unique properties make it interesting and suitable for both fundamental research and future applications. Due to its large specific area and ultra-strong absorbability, surface-functionalized graphene or graphene oxide is now used for glycan enrichment. Zhang et al. recently introduced a rapid, highly efficient, and visual approach for glycan enrichment using 1-pyrenebutyryl chloride functionalized graphene oxide [46], illustrated in Figure 5. First, graphene was functionalized by 1 -pyrenebutyric acid and following 1 pyrenebutyryl chloride treatment. Reversible covalent bonding occurs between the hydroxyl groups of the glycans and the acyl chloride groups on graphene oxide via the $\pi-\pi$ stacking of 1 -pyrenebutyryl chloride. Enrichment efficiency was much improved by the large specific surface area and heavy functionalization of active 1-pyrenebutyryl chloride. When the investigators tested this material in several sample processing applications, MS signal intensity, signal-to-noise ratio, and the number of glycoforms identified on standard oligosaccharides or the $\mathrm{N}$-glycans that were released from glycoproteins increased remarkably.

Carbon nanotubes (CNTs) are allotropes of carbon exhibiting a cylindrical nanostructure. These cylindrical carbon molecules have unusual properties that could be, and is currently, exploited by the electronics industry, optics industry, and other fields of materials science and technology. Zhang X. and colleagues constructed a novel lectin-based electrochemical biosensor of functionalized, multi-walled CNTs to capture glycans from living cells [47]. They fabricated the biosensor by adsorbing poly (diallyldimethylammonium chloride) (PDDA)-functionalized CNTs (PDCNTs) onto a glassy carbon electrode (GCE), followed by glutathione (GSH)-protected gold nanoparticles (AuNPs) adsorption. The resulting material becomes an effective platform for lectin immobilization, with high stability and bioactivity. The investigators also synthesized a thiol-derivatized carbohydrate (thiomannosyl dimer) to construct (CNT/thionine/Au-S-mannose) biocomposites, which used CNTs as carriers to load enormous amounts of thionine (to generate an electrochemical signal) and AuNPs (to anchor the thiomannosyl dimer).

The two applications for functionalized CNTs, whether as a biosensing platform and biocomposite, allow enhanced glycan signal detection. The former (electrochemical biosensor) has demonstrated good analytical performance - high sensitivity, selectivity and rapid response - for the analysis of mannose in human lung cancer cells. This approach could be adapted for other indications by broadening the selection moiety (e.g., lectins) for biosensor development.

\section{Conclusion and perspective}

Investigators have long recognized the significance of protein glycosylation in physiological processes and disease, as evidenced by the number of related publications that continue to accumulate. Furthermore, glycosylated proteins are increasing identified as biomarkers for early disease detection of diseases via proteomics methods. Although recent advances in mass spectrometry have made large-scale identification of proteins feasible, it is still very challenging to analyze protein glycosylation in complex samples owing to the fact that glycopeptides often constitute a minor portion of the total peptide mixture, whose signal intensities are often lower compared to non-glycosylated peptides and are suppressed in the presence of non-glycopeptides. Nanotechnology has undoubtedly contributed to the realization of glycoprotein/glycopeptide enrichment methods, particularly from enrichment from complex samples in preparation for MS characterization. The use of nanomaterials with various morphologies and characteristics (nanoparticles, mesoporous materials, nanosheets, nanotubes, etc.) has enabled enhanced sensitivity and specificity of glycopeptides isolation.

Challenges do remain in detecting post-translationally modified proteins/peptides, especially circulating glycopeptides. Characterizing glycosylated peptides is also difficult due to unique electrochemical properties, optical properties, and energy brand structure. By applying nanoscaled materials to tunable platforms, scientists have greatly enhanced the capabilities of traditional MS-based techniques, by increasing mass resolution, mass range, mass intensities, to name a few. We believe this significant progress blazes a trail for future efforts (and accomplishments) in the combined areas of nano-engineering, MS and glycoproteomics.

\section{Competing interests}

The authors declare that they have no competing interests.

\section{Author's contributions}

$H Z, Y L$ and $Y H$ participated the drafting of the manuscript. All of the authors read and approved the final manuscript.

\section{Acknowledgement}

We like to acknowledge DOD innovator award (DoD W81XWH-09-1-0212), NIH U54CA151668, Bill \& Melinda Gates Foundation and DoD W81XWH-11-2-0168.

Received: 29 November 2013 Accepted: 17 March 2014

Published: 13 May 2014

\section{References}

1. Tian Y, Zhang H: Glycoproteomics and clinical applications. Proteomics Clin Appl 2010, 4(2):124-132. doi: 10.1002/prca.200900161.

2. Tian $\mathrm{Y}$, Zhang $\mathrm{H}$ : Characterization of disease-associated $\mathrm{N}$-linked glycoproteins. Proteomics 2013, 13(3-4):504-511. doi: 10.1002/pmic.201200333.

3. Bruckner K, Perez L, Clausen H, Cohen S: Glycosyltransferase activity of Fringe modulates Notch-Delta interactions. Nature 2000, 406(6794):411-414.

4. Isaji T, Gu J, Nishiuchi R, Zhao Y, Takahashi M, Miyoshi E, Honke K, Sekiguchi K, Taniguchi N: Introduction of bisecting GlcNAc into integrin a $5 \beta 1$ reduces 
ligand binding and down-regulates cell adhesion and cell migration. J Biol Chem 2004, 279(19):19747-19754.

5. Zheng M, Fang H, Hakomori S-i: Functional role of N-glycosylation in alpha 5 beta 1 integrin receptor. De-N-glycosylation induces dissociation or altered association of alpha 5 and beta 1 subunits and concomitant loss of fibronectin binding activity. J Biol Chem 1994, 269(16):12325-12331.

6. Drake PM, Cho W, Li B, Prakobphol A, Johansen E, Anderson NL, Regnier FE, Gibson BW, Fisher SJ: Sweetening the pot: adding glycosylation to the biomarker discovery equation. Clin Chem 2010, 56(2):223-236.

7. Rademacher TW, Parekh RB, Dwek RA, Isenberg D, Rook G, Axford JS, Roitt I: The role of IgG glycoforms in the pathogenesis of rheumatoid arthritis. In Springer seminars in immunopathology. Springer; 1988:231-249.

8. Gebauer G, Müller-Ruchholtz W: Tumor marker concentrations in normal and malignant tissues of colorectal cancer patients and their prognostic relevance. Anticancer Res 1996, 17(4B):2939-2942.

9. Catalona WJ, Smith DS, Ratliff TL, Dodds KM, Coplen DE, Yuan JJ, Petros JA, Andriole GL: Measurement of prostate-specific antigen in serum as a screening test for prostate cancer. N Engl J Med 1991, 324(17):1156-1161.

10. Zhou L, Liu J, Luo F: Serum tumor markers for detection of hepatocellular carcinoma. World I Gastroenterol 2006, 12(8):1175.

11. Seregni E, Massimino M, Pallotti F, van der Hiel B, Cefalo G, Spreafico F, Fossati F, Bombardieri E: Serum and cerebrospinal fluid human chorionic gonadotropin (hCG) and alpha-fetoprotein (AFP) in intracranial germ cell tumors. Int J Biol Markers 2001, 17(2):112-118.

12. Simonds HM, Miles D: Adjuvant treatment of breast cancer: impact of monoclonal antibody therapy directed against the HER2 receptor. Expert Opin Biol Ther 2007, 7:487-491.

13. Alvarez-Manilla G, Warren NL, Atwood J III, Orlando R, Dalton S, Pierce M: Glycoproteomic analysis of embryonic stem cells: identification of potential glycobiomarkers using lectin affinity chromatography of glycopeptidest. J Proteome Res 2009, 9(5):2062-2075.

14. Debray H, Decout D, Strecker G, Spik G, Montreuil J: Specificity of twelve lectins towards oligosaccharides and glycopeptides related to N-glycosylproteins. Eur J Biochem 1981, 117(1):41-51.

15. Gerken TA, Revoredo L, Thome JJ, Tabak LA, Vester-Christensen MB, Clausen $\mathrm{H}$, Gahlay GK, Jarvis DL, Johnson RW, Moniz HA: The lectin domain of the polypeptide GalNAc transferase family of glycosyltransferases (ppGalNAc T's) acts as a switch directing glycopeptide substrate glycosylation in an $\mathrm{N}$-or C-Direction, further controlling mucin-type O-glycosylation. J Biol Chem 2013, 288:19900-19914

16. Tang J, Liu Y, Qi D, Yao G, Deng C, Zhang X: On-plate-selective enrichment of glycopeptides using boronic acid-modified gold nanoparticles for direct MALDI-QIT-TOF MS analysis. Proteomics 2009, 9(22):5046-5055.

17. $X u Y$, Zhang L, Lu H, Yang P: On-plate enrichment of glycopeptides by using boronic acid functionalized gold-coated Si wafer. Proteomics 2010, 10(5):1079-1086

18. Tian Y, Zhou Y, Elliott S, Aebersold R, Zhang H: Solid-phase extraction of N-linked glycopeptides. Nat Protoc 2007, 2(2):334-339.

19. Zhang H, Li X-j, Martin DB, Aebersold R: Identification and quantification of Nlinked glycoproteins using hydrazide chemistry, stable isotope labeling and mass spectrometry. Nat Biotechnol 2003, 21(6):660-666. doi: 10.1038/nbt827.

20. Zhou Y, Aebersold R, Zhang H: Isolation of N-Linked Glycopeptides from Plasma. Anal Chem 2007, 79(15):5826-5837. doi: 10.1021/ac0623181.

21. Jensen PH, Mysling S, Højrup P, Jensen ON: Glycopeptide enrichment for MALDI-TOF mass spectrometry analysis by Hydrophilic Interaction Liquid Chromatography Solid Phase Extraction (HILIC SPE). Methods Mol Biol 2013, 951:131-144

22. Liu L, Zhang Y, Zhang L, Yan G, Yao J, Yang P, Lu H: Highly specific revelation of rat serum glycopeptidome by boronic acid-functionalized mesoporous silica. Anal Chim Acta 2012, 753:64-72.

23. Hahm Jl: Biomedical detection via macro- and nano-sensors fabricated with metallic and semiconducting oxides. J Biomed Nanotechnol 2013, 9(1):1-25.

24. Fan J, Huang Y, Finoulst I, Wu H-J, Deng Z, Xu R, Xia X, Ferrari M, Shen H, Hu Y: Serum peptidomic biomarkers for pulmonary metastatic melanoma identified by means of a nanopore-based assay. Cancer Lett 2013, 334(2):202-210. doi: 10.1016/..canlet.2012.11.011. PubMed PMID: MEDLINE:23200677.

25. Bouamrani A, Hu Y, Tasciotti E, Li L, Chiappini C, Liu XW, Ferrari M: Mesoporous silica chips for selective enrichment and stabilization of low molecular weight proteome. Proteomics 2010, 10(3):496-505. doi: DOI 10.1002/pmic.200900346. PubMed PMID: ISI:000274707400012.
26. Hu Y, Bouamrani A, Tasciotti E, Li L, Liu XW, Ferrari M: Tailoring of the nanotexture of mesoporous silica films and their functionalized derivatives for selectively harvesting Low molecular weight protein. ACS Nano 2010, 4(1):439-451. doi: Doi 10.1021/Nn901322d. PubMed PMID: ISI:000273863400055.

27. Fan J, Deng XY, Gallagher JW, Huang HY, Huang Y, Wen JG, Ferrari M, Shen $\mathrm{HF}, \mathrm{Hu} Y$ : Monitoring the progression of metastatic breast cancer on nanoporous silica chips. Philos Trans R Soc A Math Phys Eng Sci 2012, 370(1967):2433-2447. doi: 10.1098/rsta.2011.0444. PubMed PMID: WOS:000302936400008.

28. Li Y, Li Y, Chen T, Kuklina AS, Bernard P, Esteva FJ, Shen H, Ferrari M, Hu Y: Circulating proteolytic products of carboxypeptidase $\mathrm{N}$ for early detection of breast cancer. Clin Chem 2013, 60:233-242. Epub 2013/10/23. doi: clinchem.2013.211953. PubMed PMID: 24146311.

29. Zheng G, Patolsky F, Cui Y, Wang WU, Lieber CM: Multiplexed electrical detection of cancer markers with nanowire sensor arrays. Nat Biotechnol 2005, 23(10):1294-1301. doi: 10.1038/nbt1138.

30. Chen RJ, Bangsaruntip S, Drouvalakis KA, Kam NWS, Shim M, Li Y, Kim W, Utz PJ, Dai H: Noncovalent functionalization of carbon nanotubes for highly specific electronic biosensors. Proc Natl Acad Sci 2003, 100(9):4984-4989.

31. Dorfman A, Kumar N, Hahm J: Nanoscale ZnO-enhanced fluorescence detection of protein interactions. Adv Mater 2006, 18(20):2685-2690. doi: 10.1002/adma.200502616.

32. Ongay S, Boichenko A, Govorukhina N, Bischoff R: Glycopeptide enrichment and separation for protein glycosylation analysis. J Sep Sci 2012, 35(18):2341-2372. doi: 10.1002/jssc.201200434.

33. Zhou W, Yao N, Yao G, Deng C, Zhang X, Yang P: Facile synthesis of aminophenylboronic acid-functionalized magnetic nanoparticles for selective separation of glycopeptides and glycoproteins. Chem Commun 2008, 43:5577-5579. doi: 10.1039/b808800d.

34. Zhang Y, Kuang M, Zhang L, Yang P, Lu H: An accessible protocol for solid-phase extraction of $\mathrm{N}$-linked glycopeptides through reductive amination by amine-functionalized magnetic nanoparticles. Anal Chem 2013, 85(11):5535-5541. doi: 10.1021/ac400733y.

35. Zhang L, Xu Y, Yao H, Xie L, Yao J, Lu H, Yang P: Boronic acid functionalized core-satellite composite nanoparticles for advanced enrichment of glycopeptides and glycoproteins. Chem A Eur J 2009, 15(39):10158-10166.

36. Xiong Z, Qin H, Wan H, Huang G, Zhang Z, Dong J, Zhang L, Zhang W, Zou $\mathrm{H}$ : Layer-by-layer assembly of multilayer polysaccharide coated magnetic nanoparticles for the selective enrichment of glycopeptides. Chem Commun 2013, 49(81):9284-9286. doi: 10.1039/c3cc45008b.

37. Tran TH, Park S, Lee H, Park S, Kim B, Kim O-H, Oh B-C, Lee D, Lee H: Ultrasmall gold nanoparticles for highly specific isolation/enrichment of N-linked glycosylated peptides. Analyst 2012, 137(4):991-998.

38. Tsutsumi H, Ohkusa H, Park H, Takahashi T, Yuasa H, Mihara H: Gold nanoparticles conjugated with monosaccharide-modified peptide for lectin detection. Bioorg Med Chem Lett 2012, 22(22):6825-6827. doi: 10.1016/.j.bmcl.2012.09.051.

39. Ma W-F, Li L-L, Zhang Y, An Q, You L-J, Li J-M, Zhang Y-T, Xu S, Yu M, Guo J, Lu $\mathrm{H}-\mathrm{J}$, Wang C-C: Ligand-free strategy for ultrafast and highly selective enrichment of glycopeptides using Ag-coated magnetic nanoarchitectures. J Mater Chem 2012, 22(45):23981-23988. doi: 10.1039/c2jm35196j.

40. Rouquerol J, Avnir D, Fairbridge C, Everett D, Haynes J, Pernicone N, Ramsay $J$, Sing K, Unger K: Recommendations for the characterization of porous solids (Technical Report). Pure App/ Chem 1994, 66(8):1739-1758.

41. Xu Y, Wu Z, Zhang L, Lu H, Yang P, Webley PA, Zhao D: Highly specific enrichment of glycopeptides using Boronic acid-functionalized Mesoporous silica. Anal Chem 2008, 81(1):503-508. doi: 10.1021/ac801912t.

42. Zhang HY, Yao GP, Deng CH, Lu HJ, Yang PY: Facile synthesis of Boronic acid-functionalized magnetic Mesoporous silica nanocomposites for highly specific enrichment of glycopeptides. Chin J Chem 2011, 29(4):835-839. doi: 10.1002/cjoc.201190166. PubMed PMID: WOS:000289938000037.

43. Wan H, Yan J, Yu L, Sheng Q, Zhang X, Xue X, Li X, Liang X: Zirconia layer coated mesoporous silica microspheres as HILIC SPE materials for selective glycopeptide enrichment. Analyst 2011, 136(21):4422-4430. doi: 10.1039/clan15554g.

44. Qin H, Zhao L, Li R, Wu RA, Zou H: Size-selective enrichment of N-linked glycans using highly ordered Mesoporous carbon material and detection by MALDI-TOF MS. Anal Chem 2011, 83(20):7721-7728. doi: 10.1021/ ac201198q. 
45. Novoselov KS, Geim AK, Morozov SV, Jiang D, Zhang Y, Dubonos SV, Grigorieva IV, Firsov AA: Electric field effect in atomically thin carbon films. Science 2004, 306(5696):666-669. doi: 10.1126/science.1102896. PubMed PMID: WOS:000224756700045.

46. Zhang W, Han H, Bai H, Tong W, Zhang Y, Ying W, Qin W, Qian X: A highly efficient and visualized method for glycan enrichment by selfassembling pyrene derivative functionalized free graphene oxide. Anal Chem 2013, 85(5):2703-2709. doi: 10.1021/ac303101t.

47. Zhang $X$, Teng $Y$, Fu Y, Zhang S, Wang T, Wang C, Jin L, Zhang W: Lectinbased electrochemical biosensor constructed by functionalized carbon nanotubes for the competitive assay of glycan expression on living cancer cells. Chem Sci 2011, 2(12):2353-2360. doi: 10.1039/c1sc00562f.

doi:10.1186/1559-0275-11-21

Cite this article as: Zhao et al:: Nanotechnologies in Glycoproteomics.

Clinical Proteomics 2014 11:21.

\section{Submit your next manuscript to BioMed Central and take full advantage of:}

- Convenient online submission

- Thorough peer review

- No space constraints or color figure charges

- Immediate publication on acceptance

- Inclusion in PubMed, CAS, Scopus and Google Scholar

- Research which is freely available for redistribution 